\title{
Livestock Model Innovation under the Concept of Smart Cities
}

\author{
Javier E. Sierra ${ }^{1}$, Juan Carlos Vesga² and Boris Medina ${ }^{1}$ \\ 'Universidad de Sucre, Cra 28 \# 5-267, Sincelejo, Colombia; javier.sierra@unisucre.edu.co, \\ boris.medina@unisucre.edu.co \\ 2Escuela de Ciencias Basicas Tecnologia e Ingenieria (ECBTI), Universidad Nacional Abierta y a Distancia; Carrera 27 \\ No.40-43. Bucaramanga, Colombia; juan.vesga@unad.edu.co
}

\begin{abstract}
Objectives: In this paper, we propose a model of livestock innovation lab employs the concept of living in the context of smart cities, which will improve the productivity of livestock farms of Sucre - Colombia. Methodology: living lab concept is analyzed and defined as it applies to cattle farms taking into account the concept of co-creation, exploration, experimentation and evaluation.Findings: A model of innovation livestock incorporating information technology and communications, implements a living lab including an information system, smart irrigation system with adequate knowledge management and decision support through big data. Application/Improvements: The model will improve livestock productivity using intelligent decision making using the concept of big data.
\end{abstract}

Keywords: 3D-Porous Coated Scaffolds, Bionanocomposite, Implantation, Non-Seeded, Rabbits

\section{Introduction}

Livestock production is one of the bests of the Department of Sucre-Colombia. This region is affected by climate variability, low production chain, low levels of value added, the security situation reflected in the theft of cattle, the fluctuating price of inputs and outputs and poor sanitary management of livestock, which emphasizes the difficulty of access to national and international markets are highly competitive and require compliance with quality standards and animal traceability. Only $2.4 \%$ of the cattle slaughtered in the Department of Sucre reach international markets ${ }^{1}$. Additionally, benefits the Information Technology and Telecommunications (ICT) under the concept of smart cities $\stackrel{2}{2}$. For the development of innovation models that contribute to the productive sector, they have not been used by the agricultural sector in Colombia, particularly livestock sector by the Department of Sucre.

The livestock sector requires the implementation of innovative strategies to increase productivity and competitiveness so that it can compete in national and international markets. Likewise, the sector can integrate the concept of living lab within their production processes that incorporate innovative models to improve knowledge management to foster the development of economies profitable and sustainable through livestock ${ }^{3}$.

This article proposes a model to develop and implement a system of innovation and knowledge management to boost quality, productivity and competitiveness of livestock Sucre Department - Colombia, under the principle of the Smart Cities ${ }^{4}$. The model employs the concept of living lab, which includes a system of information management by using ICT enabling knowledge management of the modernization of livestock processes, traceability, breeding, reproduction, nutrition and food, animal health and new agro-industrial products, production systems for milk and beef, based on the concept of quality, developing processes of science, technology and innovation in production linked to the system components.

*Author for correspondence 


\section{Materials and Methods}

\subsection{Living Lab}

A Living Lab is a new innovation model where all stakeholders (government, productive sector, academia, civil society organizations and citizens) participate actively appropriating innovation (open and collaborative), in cocreation and validation of the solutions they need them themselves in contexts of actual use, using Information Technology and Communications -TIC as a medium, thus forming an ecosystem of research and development, permanently enabling the Social Innovation $\frac{5,6}{5}$. The Living Lab is based on four main activities ${ }^{7}$ :

- Co-creation: co-design users and producers,

- Exploration: discovering emerging usages, behaviors and market opportunities,

- Experimentation: The implementations of scenarios "live" within communities of users, and

- Assessment: evaluation of concepts, products and services according to socio-cognitive and socioeconomic criteria.

The living lab aims through the implementation of cocreation generate new agro-industrial products and technological developments to innovate in the livestock process, developing knowledge management for a competitive productive livestock, to generate economic and social value through the development of the following strategic priorities, related to the implementation of the productive system:
- Reconversion: incorporating higher added value, diversify agricultural production and improve production of goods and environmental services,

- Science technology and technical assistance: generating value added to the product,

- Modifying decisions and behavior of people,

- Information and knowledge management: support decision-making for rural agricultural planning processes articulated productive system, and

- Tracking, monitoring and evaluation of management plans productive.

\subsection{Livestock Living Lab}

Model design starts with the characterization of livestock Sucre Department; including an analysis of the geography where cattle farms are located which allow the system decision count on real data from the location of each herd.

The innovation model of farming system, from the cocreation with the participation of farmers, association of farmers, society and team project work (experts in different areas), can be determined new services and products that can be developed in each herd. The process is part of analyzing problems through brainstorming sessions, focus groups, interviews and surveys, which allow generate ideas which can then be co-designed. Figure 1 shows the process and the proposed phases of living lab.

Each phase is executed by the computer innovation, analyzing the co-designs and performing step scanning which seeks to make a rapid prototyping or development of process/product. Once the prototype is / process / prod-

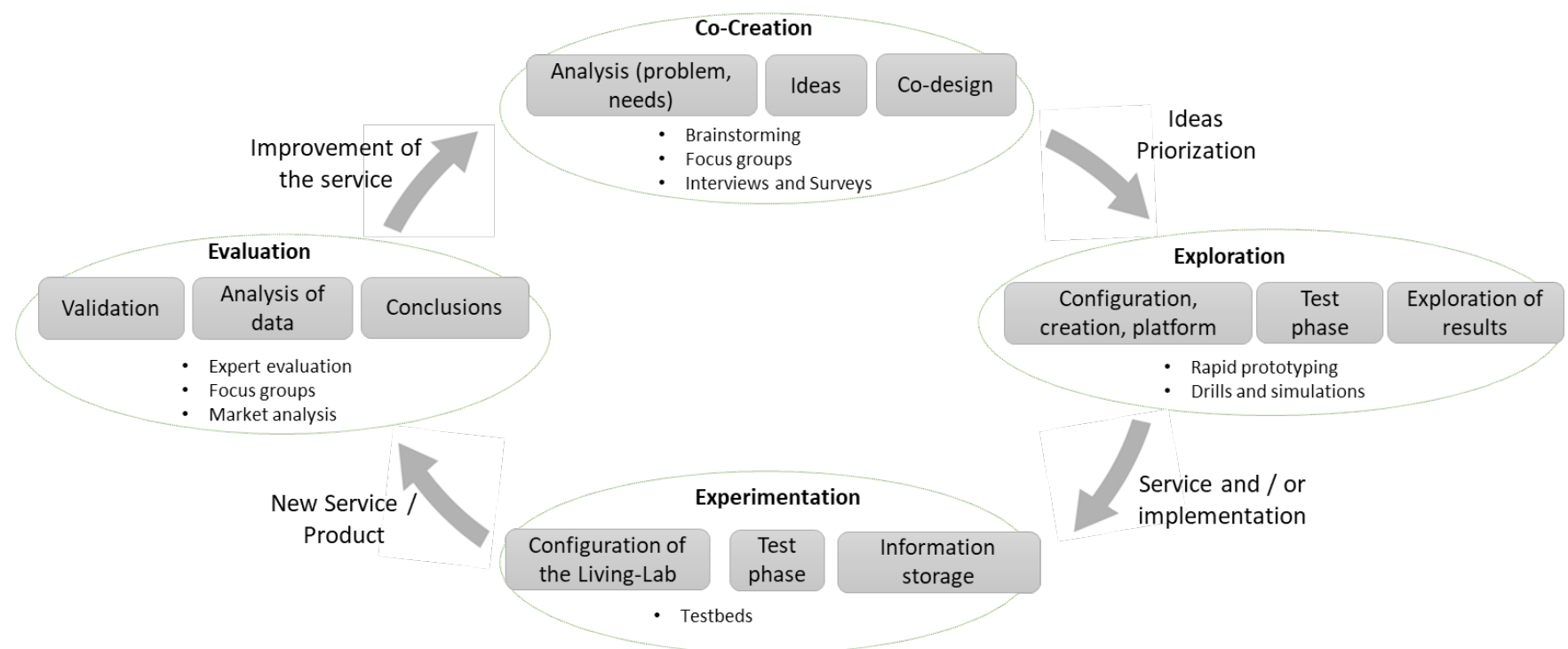

Figure 1. Process and phases of living lab proposed. 
uct are brought back to the herd to determine through experimentation the impact. The process is again assessed through focus group. The process of co-creation will allow the development of each of the activities adapted to the requirements of each cattle farm, as each sub-department has different characteristics.

\section{Results}

\subsection{Proposed Model}

The livestock sector of the Department of Sucre presents different problems, which lead to low productivity and can be grouped in three areas corresponding to the lack of control herd on farms, lack of efficient quality control at the institutional level and lack of a system for decisionmaking with relevant local and regional level information. The proposed model incorporates the Living Lab shown in Figure 2 in order to manage information and thus solve the various problems mentioned.

\subsection{Incorporation of ICT under the Concept of Smart Cities}

Cities in their planning processes should display the incorporation of ICT in their growth and development models in order to generate these different technological and social innovations that allow them to be sustainable in a globalized world. The concept used for cities that incorporate ICT in their city model called Smart City 2 . In the Smart City can innovate creating technological developments capable of managing different fields, such as mobility (smart mobility), agro electricity (smart grid), safety, environment, others.

An intelligent city is one that is characterized by intensive use of ICT in the creation and improvement of the systems that make up the city (infrastructure, energy, agriculture, health, transport, traffic, etc.), as well as their ability to create, collect, process and transform information so that their processes and services are increasingly better and more efficient, allowing to improve the quality of life of citizens through the efficient use of resources (sustainability) ${ }^{\circ}$.

Internet of Things (IoT) is a paradigm of recent communication referred to in the objects of everyday life will be equipped with microcontrollers, sensors, transceivers for digital communication, and a set of protocols that enable communication near future between yes and users, becoming an integral part of Internet ${ }^{9}$. IoT basically is the convergence of two technologies: the Internet and sensor networks (sensor networks), this creates new possibilities, allowing among others, direct communication machinemachine on the Internet. The paradigm has allowed researchers to develop and make proposals on sustainable smart cities (autonomous networks and services, etc.). Given this, it enables easy access and interaction of a wide variety of devices, such as appliances, surveillance

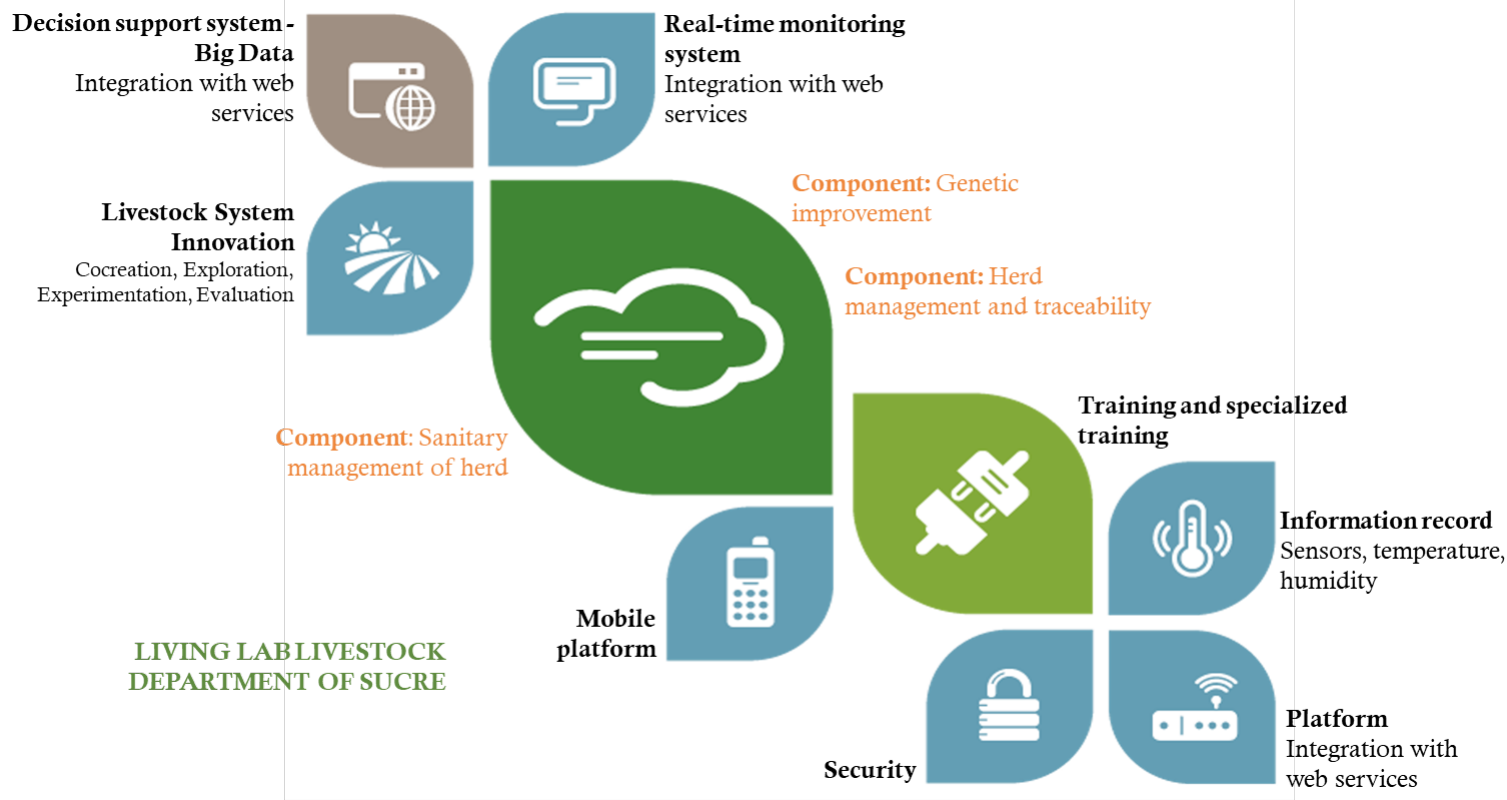

Figure 2. Living lab system livestock department of Sucre. 
cameras, sensors, actuators, displays, vehicles, others, generating an amount of information $\frac{10}{}$.

The concept of Smart City represents an attractive platform for service innovation IoT, where service providers, public and private companies use information technology to engage citizens and create more effective urban organizations and systems that can improve quality of life ${ }^{-}$. IoT is why it is essential for the development of smart cities, where integrated cloud-oriented architectures, software, sensors, human interfaces, and data analysis are essential for creating added value.

\subsubsection{Herd Information System}

Using a commercial software systematization of cattle herds optimizes and facilitates management of this administration, helping to get organized, clear and accurate reports that may be helpful in making decisions, for better production and market competitiveness. It also allows changing the design by employees to the concept of entrepreneurship, making a commitment to have more serious and responsible company, which will provide a suitable working environment.

Using software aims to ensure proper handling of information, allowing for updated and accurate for timely decision-making reports. In this sense, the implementation of information systems in herds' farmers may facilitate animal handling and allow evaluation of each of the factors involved in production, such as nutrition, reproduction, health and component human, and the interaction between them.

The software is very important for information management tool because it provides data for each animal and herd generally easy, complete and detailed way. Farms will be systematized in order to get the most complete, organized and available from herd information, decreasing the maximum use of cards and notebooks, in order to preserve better the information and make a professional job and corporate character.

When software is used it is helpful for the owner of the herd and much more for a company that needs accurate data and information for making timely decisions and improve profitability by more productive animals, increasing the economy of the company. Therefore, the formalization of the property and the business mentality that seeks to instill in all attached to herd personnel, allows the development of the people involved, making them feel part of a reputable company and projection into the future, they ensure to some extent an economic and job stability, which will bring an undeniable increase in the quality of life of each.

\subsubsection{Intelligent and Technifield Irrigation Systems for Small Plots}

The challenges to face the consequences of impending climate change on farms or small plots (less than 20Ha) in the Colombian Caribbean region are important and require urgent action, since, in most cases, do not have sufficient financial resources to respond commercial technology to such effects.

The resilience of the large farms, the core activity is livestock, is not comparable to small plots. For this reason, it is necessary to rethink irrigation technology and adapt it to the specific needs for small plots, i.e. adapting and appropriating technology both water treatment and distribution in the areas of culture, meeting the specific requirements of each seedling and forage species that are intended to benefit.

Among the predominant technologies for irrigation are drip systems, portable sprinkler systems and family systems for pasture. However, they not designed with smart water management systems based on the characteristics of each day and hourly weather conditions, representing real waste water increasingly scarce and difficult to store in small plots.

It intends to propose design criteria in the design of irrigation small scale depending on the characteristics of each herd and their problems, addressing the following objectives:

- Design, build and operate a system for smart irrigation to a smaller scale culture,

- Establish criteria for economic and technological adaptability of irrigation systems, according to particular conditions of crops and not only the hydraulic conditions prevailing on the premises, and

- Ask autonomous and intelligent irrigation systems for crops from solar energy.

Among the advantages to be achieved with this model they are:

- Achieve ease and practicality in the installation, operation and maintenance of smart irrigation systems,

- Increase the yield and quality of irrigation in farming areas, 
- Greater efficiency in water use,

- Economy in use of labor,

- Elimination of the need for use of electricity and fuel,

- Avoid wetting the foliage in phytosanitarily sensitive crops,

- Allowing the possibility of fertigation, and

- Prevent soil erosion.

\subsubsection{Develop and Implement the Management Model of Knowledge through ICT}

The implementation of technologies in farms allows data collection quickly and timely, in order to be used at any time for analysis of reproductive, productive, genetic, health and cost parameters. The main objective of this component is to implement a technological tool that allows the farmer to optimize its production system (meat and / or milk) in order to have at all times records different parameters for decision-making and avoid the use of paper.

\subsubsection{Process Training and Operation of the Platform}

The training process is one of the main elements of the model, proposing to do it in the municipalities where they are located cattle herds with a thematic expert innovation team, who will: training, ownership and implementation on devices that each recipient available for the same.

Training and appropriation are processes through which tools for beneficiaries are aware of the importance of the processing of production records to be efficient and competitive in the market, avoid loss of money for predictable omissions and make planning will be given strategies and activities through the use of information that can be obtained from the herd.

\subsection{Decision Support through Big Data}

To achieve efficient management model innovation, production optimization and competitiveness of herds of livestock in Sucre, it intends to put into operation a computing platform under the concept of big data [eleven] which would connect all variables and actors that are part of the Living Lab. The information platform will control the process and information, product it in each of its phases. The following are the features to provide the information system:
- Information system user friendly interface, easy to use and intuitive; streamlining daily tasks management information to be entered and modified easily,

- The information must be updated in real time and available on all your devices,

- Information stored in the cloud, $100 \%$ web,

- Each user must have a profile with a password that allows you, according to your level of authorization to obtain information from the platform or process and record activities,

- It ensures traceability and reliability of information,

- The system has a suite of reports; tabula, collects and organizes the project data and transforms them into rich visual objects, allowing you to analyze data and share information, and lets you focus on what really matters, and

- Users can perform a scan 360 degrees with your most important metrics in one place.

The proposed system can obtain, for example, information each, resume and technical specifications, traceability of updated and detailed information herd productive. The analyses of process indicators allow you to make tight against the herd decisions:

- Genetically selected number of copies,

- Possible examples for replacement,

- Genetic value inherited in obtained offspring,

- Weight gain in calves,

- Increased liters of milk produced, and

- Agro industrial new products.

\subsection{Technology Transfer that allows Management to Implement Prototypes and Modernization of Cattle Farms}

A given level of technology and the concept of Internet of Things are different possible transfers of technologies that could be developed in each of the cattle farms. The initial concept to be implemented is that all farms will be connected to the Internet so they can report different variables to system management information. Among these variables are: temperature, humidity, solar radiation level, soil moisture, precipitation \%, level of water reserves, other.

In applying the model of co-creation in each of the farms, you can determine how the prototype, so it is pos- 
sible to obtain innovation processes in the herd will be implemented. It has considered the possibility of transferring:

- Mobile application with information concerning the cattle ranch that allows the user to access information in real time on his farm,

- Management of water supplies and irrigation automation,

- remote station physical data acquisition of the property (moisture, temperature, rainfall, climatic variations),

- Implementation of alternative systems that allow power generation sustaining equipment installed,

- Physical traceability of cattle through RFID devices, and

- Geolocation of each herd with all its features.

All these possible prototypes will feed the information system, which will seek to keep all information in time so that possible.

\section{Conclusions}

This article proposes a model of farmer innovation, which uses the concept of living lab. The model incorporates phase's co-creation, exploration, experimentation and evaluation through an information system that includes variables and characteristics of cattle herds for continuous improvement and generating new livestock products. The model improves the productivity of livestock sector, since the decision system is supported on big data.

\section{References}

1. FEDEGAN. Federación Colombiana de Ganaderos, Informe De Gestión, Vigencia; 2014. p. 293. Date accessed: 01/05/2010. http://static.fedegan.org.co/ Ley_1712/01_Informes_de_Gestion_y_Plan_Estrategico/ Informe_de_Gestion_Consolidado_2014.pdf.

2. Chun BT. A study on analysis and applicability of current Smart City, Indian Journal of Science and Technology. 2015; 8(S7):314-19. Crossref.

3. Liu G, Liu P, Zhang Q. Ecological and Socio-Economic Benefits of Investment in an Environmental Restoration Program and Agricultural Management in China. In: 4th International Conference on Bioinformatics and Biomedical Engineering; 2010. p. 1-4. Crossref.

4. Madakam S, Ramaswamy R. Sustainable Smart City: Masdar (UAE) (A City: Ecologically Balanced), Indian Journal of Science and Technology. 2016; 9(6):1-8. Crossref.

5. Ramesh MV, Mohan R, Menon S. Live-in-Labs: Rapid translational research and implementation-based program for rural development in India. In: IEEE Global Humanitarian Technology Conference (GHTC); 2016. p. 164-71. Crossref.

6. Elisabeth S, Rossi A, Bertoldi F, Fusco L, Santoro R. Collaborative effort in the Frascati Living Lab.IEEE International Technology Management Conference (ICE); 2008. p. 1-8.

7. Introducing ENoLL, the European Network of Living Labs. Date accessed: 10/03/2014. https://pro.europeana.eu/post/ introducing-enoll-the-european-network-of-living-labs.

8. Harmon RR, Castro-Leon EG, Bhide S. Smart cities and the Internet of Things. In Portland International Conference on Management of Engineering and Technology (PICMET); 2015. p. 485-94. Crossref.

9. Whitmore A, Agarwal A, Xu LD. The Internet of Things-A survey of topics and trends, Information Systems Frontiers. 2015; 17(2):261-74. Crossref.

10. Schaffers H, Komninos N, Pallot M, Trousse B, Nilsson M, Oliveira A. Smart Cities and the Future Internet: Towards Cooperation Frameworks for Open Innovation. The Future Internet Assembly, Springer, Berlin, Heidelberg; 2011. p. 431-46. 